\title{
Perfil químico y actividad antioxidante de aceites esenciales de hierbas aromáticas altoandinas del Perú
}

\author{
Chemical profile and antioxidant activity of the essential oil of native high Andean species of Perú
}

Perfil químico e atividade antioxidante do óleo essencial de espécies nativas dos altos Andes do Perú

\author{
Rosa Huaraca Aparco \\ rhuaraca@unajma.edu.pe \\ https://orcid.org/0000-0003-4493-7754 \\ Fidelia Tapia Tadeo \\ ftapia@unajma.edu.pe \\ https://orcid.org/0000-0003-4892-941X
}

\author{
María Del Carmen Delgado Laime \\ mcdelgado@unajma.edu.pe \\ https://orcid.org/0000-0002-7911-8647
}

\author{
Guido Nolasco Carbajal \\ gnolasco@unajma.edu.pe \\ https://orcid.org/0000-0002-5472-8254
}

\section{Universidad Nacional José María Arguedas, Andahuaylas, Perú}

\section{RESUMEN}

Las hierbas aromáticas tienen una amplia distribución en la región de Apurímac, Perú, donde encontramos especies silvestres y cultivadas, su interés se basa en la presencia de aceites esenciales que contiene componentes bioactivos que son usados como conservantes en la industria de alimentos. El objetivo de la investigación fue determinar el perfil químico y actividad antioxidante de los aceites esenciales de Lepechinia meyenii (Walp) Epling y Mentha piperita L. La extracción de aceites esenciales se realizó mediante la destilación por arrastre de vapor de agua a condiciones de sobresaturación. Se determinaron los siguientes parámetros: rendimiento de extracción, densidad relativa e índice de refracción. El perfil químico se realizó mediante la cromatografía de gases acoplada a espectrómetria de masas y la actividad antioxidante mediante la eliminación del radical libre 1,1-difenil-2-picrilhidrazilo y H2O2. (DPPH), como resultado, el porcentaje de rendimiento fue del $0,87 \%$ y $0,56 \%$, con densidades de 0,942 g/mL y 0,919 g/mL y índice de refracción de 1,4917 y 1,4676 para Lepechinia meyenii (Walp) Epling y Mentha piperita L.), respectivamente. Los aceites esenciales se caracterizaron principalmente por una fracción sesquiterpenoides en un $(55,73 \%)$ y con una fracción monoterpenoides en un (55,60\%). La actividad antioxidante fue significante de 4,95-0,06 mg/mL y 7,21-0,21 mg/ $\mathrm{mL}$. Los aceites esenciales a partir de hierbas aromáticas presentaron significativamente altos componentes químicos y actividad antioxidante, siendo posibles candidatos antimicrobianos, antioxidantes, antibacterianas para la industria alimentaria.

Palabras clave: Aceite esencial; actividad antioxidante; cromatografía, hierbas aromáticas; perfil químico
ABSTRACT

Aromatic herbs have a wide distribution in the Apurímac region, Peru, where we find wild and cultivated species, their interest is based on the presence of essential oils that contain bioactive components that are used as preservatives in the food industry. The objective of the research was to determine the chemical profile and antioxidant activity of the essential oils of Lepechinia meyenii (Walp) Epling and Mentha piperita L. The extraction of essential oils was carried out by means of water vapor distillation under conditions of supersaturation. The following parameters were determined: extraction yield, relative density and refractive index. The chemical profile was made by gas chromatography coupled to mass spectrometry and the antioxidant activity by elimination of the free radical 1,1-diphenyl2-picrylhydrazyl and $\mathrm{H} 2 \mathrm{O} 2$. (DPPH), as a result, the yield percentage was $0.87 \%$ and $0.56 \%$, with densities of $0.942 \mathrm{~g} / \mathrm{mL}$ and $0.919 \mathrm{~g} / \mathrm{mL}$ and refractive index of 1.4917 and 1.4676 for Lepechinia meyenii (Walp) Epling and Mentha piperita L.), respectively. Essential oils are mainly characterized by a sesquiterpenoid fraction (55.73\%) and a monoterpenoid fraction (55.60\%). The antioxidant activity was significant at 4.95-0.06 mg / mL and 7.21-0.21 mg / mL. Essential oils from aromatic herbs presented high chemical components and antioxidant activity, being possible antimicrobial, antioxidant, antibacterial candidates for the food industry.

Key words: Essential oil; antioxidant activity; chromatography, aromatic herbs; chemical profile

\section{RESUMO}

As ervas aromáticas têm ampla distribuição na região de Apurímac, Peru, onde encontramos espécies silvestres e cultivadas, seu interesse se baseia na presença de óleos essenciais que contêm componentes bioativos que são usados como conservantes na indústria alimentícia. O objetivo da pesquisa foi determinar o perfil químico e a atividade antioxidante dos óleos essenciais de Lepechinia meyenii (Walp) Epling e Mentha piperita L. A extração dos óleos essenciais foi realizada por meio de destilação a vapor d'água em condições de supersaturação. Os seguintes parâmetros foram determinados: rendimento de extração, densidade relativa e índice de refração. O perfil químico foi feito por cromatografia gasosa acoplada a espectrometria de massas e a atividade antioxidante por eliminação dos radicais livres 1,1-difenil-2-picrilhidrazila e $\mathrm{H} 2 \mathrm{O} 2$. (DPPH), como resultado, o percentual de rendimento foi de $0,87 \%$ e $0,56 \%$, com densidades de 0,942 g / mL e 0,919 g / mL e índice de refração de 1,4917 e 1,4676 para Lepechinia meyenii (Walp) Epling e Mentha piperita L.), respectivamente. Os óleos essenciais são caracterizados principalmente por uma fração sesquiterpenóide $(55,73 \%)$ e uma fração monoterpenóide (55,60\%). A atividade antioxidante foi significativa em 4,95-0,06 $\mathrm{mg} / \mathrm{mL}$ e 7,21-0,21 mg / mL. Os óleos essenciais de ervas aromáticas apresentam elevada atividade antioxidante e química, sendo possíveis candidatos antimicrobianos, antioxidantes e antibacterianos para a indústria alimentícia.

Palavras-chave: Óleo essencial; atividade antioxidante; cromatografia, ervas aromáticas; perfil químico 


\section{INTRODUCCIÓN}

Perú es uno de los 12 países con la mayor diversidad biológica, con aproximadamente $10 \%$ de flora mundial, estimada en 2500 especies, 30 de ellas son endémicas (1). El interés por los componentes activos de plantas nativas como alternativa al uso de compuestos químicos han incrementado las investigaciones (2). Los aceites esenciales son líquidos hidrofóbicos concentrados aromáticos y volátiles obtenidos de las plantas, están distribuidos por monoterpenoides, sesquiterpenodes, alcoholes, ácidos, esteres acíclicos aldehídos y lactonas (3). Los aceites esenciales reciben atención por su actividad antimicrobiana contra diferentes tipos de bacterias y hongos (4). Citostática e insecticida también se usan como flavores alimentarios (5), como aditivos naturales en alimentos (6). Los compuestos fenólicos presentes en los aceites esenciales les otorgan sus propiedades antioxidantes (7). El origen geográfico, nivel de precipitación y condiciones climáticas son los aspectos más importantes que influyen en la composición de los aceites esenciales (8-10). La creciente demanda por alternativas naturales a los aditivos sintéticos ha impulsado la búsqueda de nuevas fuentes principalmente vegetales (11), en alimentación, es muy importante su capacidad conservante (12), y para controlar la calidad de los aceites se evalúa generalmente la gravedad especifica (GE) eíndice de refracción (IR)como indicadores $(13,14)$. La evaluación de la seguridad de un $A E$ involucra la especificación de su origen biológico, propiedades físicas y químicas y cualquier otra característica relevante (15). El género Lepechinia de la familia Lamiaceae está compuesta de 40 a 42 especies distribuidas desde el sur de Chile hasta California, USA. Lepechinia salviae (Lindl.) Epling, es una de las tres especies endémicas de Lepechinia presentes en Perú junto con Lepechinia subhastata (Benth) Epling. En algunos informes se identifica por el nombre sinónimo Sphacele salviae $(16,17)$.

Entre los componentes activos reportados en la especie Lepechinia salviae fueron; compuestos fenólicos como carnosol, rosmanol, ácido carnosico y 20 desoxocarnosol fueron los compuestos principales que le dieron a esta planta actividad antioxidante $(16,18)$ y antimicrobiana (4). Esta especie se utiliza para tratamiento de afecciones del estómago, faringe, hígado, refriados (19). Nuestro interés en el género Lepechinia se debe a la abundancia de aceites esenciales y la variedad de metabolitos secundarios producidos (20). La Mentha peperita L. es un hibrido natural cultivado de Mentha aquatica L. y Mentha spicata L. aunque es originario de la región mediterránea, se cultiva en todo el mundo por su aceite esencial de menta se utiliza en sabor, fragancia, medicinal y aplicaciones farmacéuticas $(21,22)$. Se han identificado más de 300 componentes en el aceite esencial donde los componentes son mentol, mentona, acetato de mentilo, 1,8 cineol, mentofurano, isomentona y limonerno $(23,24)$ a pesar de que el potencial medicinal de las plantas en el Perú es considerable, el conocimiento de esta área y los estudios sobre las actividades biológicas de estas plantas siguieron siendo escasas. De acuerdo a antecedentes investigativos los componentes químicos y actividad antioxidantes de las plantas aromáticas peruanas Tanacetum vulgare L. y Mentha piperita L. no se han publicado previamente sin embargo habiendo muchos informes sobre aceites esenciales de estas especies en otros países. A pesar de su importancia como hierbas aromáticas altoandinas y 
especies medicinales, las investigaciones en especies Lepechinia y Mentha con respecto a su composición químicas diversidad genética y propiedades biológicas son limitadas. Por lo tanto, el objetivo del presente estudio ha sido determinar la composición química de los aceites esenciales y las actividades antioxidantes de ambas hierbas aromáticas altoandinas que crecen a gran altura de la región andina del Perú.

\section{MATERIALES Y MÉTODOS}

\section{Materia Vegetal e identificación botánica}

Se utilizaron las hojas de Lepechinia meyenii (Walp) Epling y Mentha piperita L. recolectadas de la zona alto ndel distrito de José María Arguedas ( $13^{\circ} 42 \mathrm{~S} .73^{\circ} 24 \mathrm{O}$ a una altitud de $2935 \mathrm{msnm}$ ) perteneciente a la provincia de Andahuaylas, región Apurímac. Con clima Cwd de acuerdo a Koppens con precipitaciones media anual alrededor de $1000 \mathrm{~mm} / \mathrm{año}$, humedad relativa media de 50 $\%$ y temperatura de $-5^{\circ} \mathrm{C}$ a $21^{\circ} \mathrm{C}$, con moderada incidencia de heladas. Las hojas de Lepechinia meyenii (Walp) Epling y Mentha piperita L. fueron cosechadas durante los meses de febrero a marzo del 2019. Las plantas fueron identificadas y autentificadas por la Dra. María del Carmen Delgado Laime y depositada en el laboratorio de botánica del pabellón de Ciencias Básicas de la Universidad Nacional José María Arguedas.

\section{Extracción de aceites esenciales}

Para la extracción de los aceites esenciales se seleccionaron las hojas frescas de Lepechinia meyenii (Walp) Epling y Mentha piperita L; se utilizaron 2,5 kilogramos de hojas frescas de cada especie y se sometieron a extracción por destilación por arrastre a vapor de agua a una presión de 10 psi. Una vez destilado los aceites esenciales se separaron por diferencia de densidades utilizando un decantador florentino graduado. Luego se secaron sobre sulfato de sodio anhidro y se almacenó a $4{ }^{\circ} \mathrm{C}$ hasta el análisis, los rendimientos de extracción se evaluaron según la ecuación 1.

$\% P=\frac{\text { Masa final de aceite esencial }(g)}{\text { Masainicial de muestra o follaje }(g)} * 100$

\section{Determinación de densidad relativa}

La densidad relativa fue determinada de acuerdo a la norma técnica peruana: NTP 3129.081:1974. Se calculó la densidad relativa de acuerdo a la ecuación 2.

$\mathrm{DR}=(\mathrm{P}$ picnometro + muestra $)-($ Ppicnometro $) \frac{(g)}{V A B}(m L)$

Donde:

Picnómetro: peso del picnómetro

VAE: volumen del aceite esencial.

\section{Determinación del Índice de refracción}

Se utilizó el refractómetro ABBE, marca Ivymen System, Modelo RI-71, mediante la Norma Técnica Peruana: NTP 319, 075:1974.

\section{Determinación de compuestos químicos mediante cromatografía de gases acoplada a espectrometría de masas (GC-MS)}

Para el análisis de cada muestra se utilizó $20 \mu \mathrm{L}$ de aceite esencial en $980 \mu \mathrm{L}$ de diclometano, que fueron después de inyectar al cromatógrafo de gases acoplado a un detector selectivo de masas. Se separaron los compuestos en una mezcla mediante una columna capilar apolar DB-5MS $(60 \mathrm{~m}$ x250 $\mu \mathrm{m} \times 0,25 \mu \mathrm{m})(\mathrm{J}$ y W Scientific de $5 \%$ fenil-polimetilsiloxano). La temperatura del inyector se mantuvo a $250^{\circ} \mathrm{C}$ con una inyección 
en modo Split (50:1), la programación de temperatura del horno fue: temperatura inicial $50^{\circ} \mathrm{C}$, mantenida por 5 minutos; posteriormente incrementándose a $10{ }^{\circ} \mathrm{C} / \mathrm{min}$ hasta alcanzar $100{ }^{\circ} \mathrm{C}$ y finalmente a $10{ }^{\circ} \mathrm{C} / \mathrm{min}$ hasta $270{ }^{\circ} \mathrm{C}$, manteniéndose temperatura final por $1 \mathrm{~min}$. $\mathrm{El}$ tiempo de corrida fue de 77,8 minutos, se utilizó helio como gas de arrase a un flujo constante de $1 \mathrm{~mL} / \mathrm{min}$. Los compuestos de los aceites de Lepechinia meyenii (Walp) Epling y Mentha piperita $\mathrm{L}$ fueron identificados utilizando el software proporcionado por Agilent; MSD chemstation (versoon EO2.00.493), Por comparación de los espectros de masas de cada pico con los de la librería de espectros de masas de las bases de datos de flavor 2 y del Instituto Nacional de Estándares y Tecnología (NIST, 08).

\section{Determinación de la actividad antioxidante}

Se prepararon diluciones en etanol acuoso de extractos hidroalcohólicos hasta obtener concentraciones de 0,0 a 150,0 $\mu \mathrm{g} / \mathrm{mL}$. Se combinaron $1,0 \mathrm{~mL}$ de cada dilución con 0,5 $\mathrm{mL}$ de una solución de 0,3 mM de $\mathrm{DPPH}$ en etanol dejándose reaccionar a temperatura ambiente por 30 minutos, posteriormente se llevó a medir la absorbancia de las mezclas a $517 \mathrm{~nm}$ en el equipo de espectrofotometría. El porcentaje de actividad antioxidante de cada muestra se calculó de acuerdo a la siguiente ecuación 3:

Actividad antioxidante $(\%)=\frac{A C-A M-A B}{A C} \times 100$

Donde:

AM: es la absorbancia de la muestra + DPPH

AB: es la absorbancia del blanco (muestra +etanol)

AC: es la absorbancia del blanco del reactivo (DPPH+etanol)
La concentración del extracto hidroalcohólico se neutralizó al 50 por ciento de los radicales de DPPH (EC50, concentración efectiva media) y se obtuvo directamente al graficar la recta entre el porcentaje de actividad antioxidante, frente a la concentración de la muestra de los aceites esenciales $\mathrm{mg} / \mathrm{mL}$.

\section{Análisis estadístico}

Los análisis muéstrales se realizaron por triplicado, para la evaluación estadística se utilizó un diseño completamente al azar (DCA); el análisis de varianza se trabajó con 0,05 de significancia; al encontrar diferencia significativa se procedió a realizar la prueba de comparaciones de medias de Fischer (LSD) a un nivel de $\alpha=0,05$. Los datos fueron procesados con la ayuda de los programas estadísticos de Centurion XVII y la hoja de cálculo Microsoft Excel 2016.

\section{RESULTADOS}

Los aceites esenciales de Lepechinia meyenii (Walp) Epling y Mentha piperita L mostraron un rendimiento del 0,87 \% y 0,56 $\%$ y la densidad relativa fue de 0,942 y 0,919 $\mathrm{g} / \mathrm{mL}$, para ambos resultados se encontró una diferencia significativa $p \leq 0,05$. El índice de refracción fue 1,4917 y 1,4676 con una diferencia significativa de $p$-value $\leq 0,05$. Se detectaron y cuantificaron un total de 61 compuestos químicos para el aceite esencial de Lepechinia meyenii (Walp) Epling, siendo los hidrocarburos sesquiterpenicos la fracción principal de $(55,73 \%)$. Los compuestos principales $(\geq 1,0 \%)$, según el orden de elución fueron $\alpha$-Pineno (1,96\%), D-Limoneno (6,48\%), Copaeno (2,06\%), a-Gurjuneno (1,34\%), $\beta$ Cariofileno (1,39\%), $\beta$ - Cariofileno (2,19\%), $\alpha$-Farneseno (3,81\%), $\beta$-Bisaboleno $(2,42 \%)$, $\alpha$-Amorfeno (2,30\%), $\delta$-Cadineno (3,63\%), 
Guaiol (16,32\%), a-Cadinol (1,30\%), $\beta$-Eudesmol (2,36\%), Bulsenol (4,15\%) y $\alpha$-Bisabolol (6,49\%). Con una desviación estándar de menos del $5 \%$ entre los porcentajes de cada analito en ambas columnas, en el total de los componentes detectados doce estaban indeterminados y se omiten de la Tabla 1.

Con respecto al aceite esencial de Mentha piperita L. se detectaron y cuantificaron 36 constituyentes, siendo los compuestos monoterpenoides principales $(\geq 1,0 \%)$;
$\beta$-Mirceno (3,15\%), $\beta$-trans-ocimeno (4,58\%), $\beta$-cis-ocimeno $(1,96 \%), \quad \beta$-Linalol $(30,25 \%)$, a-Terpineol (1,29\%), o-aminobenzoato de linalilo (27,32\%), trans-Geraniol (4,26\%), Acetato de Nerol (2,92\%) y Acetato de Geraniol $(5,48 \%)$. Se obtuvo una desviación estándar por debajo del $5 \%$ entre los porcentajes de cada analito en ambas columnas utilizadas. Siete componentes detectados están indeterminados y se omiten de la Tabla 2.

Tabla 1. Composición química del aceite esencial de Lepechinia meyenii (Walp) Epling.

\begin{tabular}{|c|c|c|c|}
\hline Número & Nombre del compuesto (NIST08.L) & $\operatorname{tR}(\min )$ & $\begin{array}{l}\% \text { en la muestra } \\
\text { áreas relativas) }\end{array}$ \\
\hline 1 & a-Pineno & 13.38 & 1.96 \\
\hline 2 & $\beta$-Pineno & 14.87 & 1.63 \\
\hline 3 & $\beta$-Mirceno & 15.01 & 0.14 \\
\hline 4 & o-Cimeno & 16.30 & 0.43 \\
\hline 5 & D-Limoneno & 16.48 & 6.48 \\
\hline 6 & Eucaliptol & 16.62 & 2.72 \\
\hline 7 & $\beta$-Linalol & 18.55 & 0.22 \\
\hline 8 & Acetato de 1-Octen-3-ilo & 18.65 & 0.35 \\
\hline 9 & Acetato de 2-Octil & 19.03 & 0.25 \\
\hline 10 & Tujona & 19.33 & 0.62 \\
\hline 11 & cis-Tagetona & 19.98 & 0.27 \\
\hline 12 & 3-Pinanona & 21.24 & 0.24 \\
\hline 13 & 4-terpineol & 21.29 & 0.20 \\
\hline 14 & Mirtenol & 21.75 & 0.89 \\
\hline 15 & Pulegona & 23.00 & 0.38 \\
\hline 16 & Acetato de trans-Pinocarvilo & 24.56 & 2.44 \\
\hline 17 & Acetato de Mirtenilo & 25.35 & 11.41 \\
\hline 18 & a-Cubebeno & 26.06 & 0.68 \\
\hline 19 & Copaeno & 26.94 & 2.06 \\
\hline 20 & a-Gurjuneno & 27.83 & 1.34 \\
\hline 21 & $\beta$-Cariofileno & 28.21 & 1.39 \\
\hline 22 & a-Bergamoteno & 28.32 & 2.19 \\
\hline 23 & $\beta$-Cubebeno & 28.43 & 0.49 \\
\hline
\end{tabular}




\begin{tabular}{|c|c|c|c|}
\hline Número & Nombre del compuesto (NIST08.L) & $\operatorname{tR}(\min )$ & $\begin{array}{l}\% \text { en la muestra } \\
\text { áreas relativas) }\end{array}$ \\
\hline 24 & (Z)- $\beta$-Farneseno & 28.63 & 0.42 \\
\hline 25 & Allo-Aromadendreno & 28.70 & 0.37 \\
\hline 26 & $\alpha$-Cariofileno & 29.17 & 0.39 \\
\hline 27 & Germacreno D & 29.29 & 0.31 \\
\hline 28 & Y-Muroleno & 29.61 & 0.66 \\
\hline 29 & (Z,E)-Farneseno & 29.75 & 0.19 \\
\hline 30 & (E)- $\beta$-Farneseno & 29.80 & 0.22 \\
\hline 31 & a-Farneseno & 30.03 & 3.81 \\
\hline 32 & Himachala-3(12),4-dieno & 30.08 & 0.25 \\
\hline 33 & a-Muuroleno & 30.16 & 0.94 \\
\hline 34 & $\beta$-Bisaboleno & 30.28 & 2.42 \\
\hline 35 & a-Amorfeno & 30.63 & 2.30 \\
\hline 36 & $\delta$-Cadineno & 30.69 & 3.63 \\
\hline 37 & Calameneno & 30.84 & 0.80 \\
\hline 38 & cis- $\alpha$-Bisaboleno & 31.13 & 0.59 \\
\hline 39 & $\begin{array}{l}{[1 \mathrm{R}-(1 \alpha, 4 a \beta, 8 \mathrm{a} \alpha)]-1,2,4 a, 5,6,8 \mathrm{a}-\mathrm{hexahidro-4,7-}} \\
\text { dimetil-1-(1-metiletil)-Naftaleno }\end{array}$ & 31.25 & 0.29 \\
\hline 40 & trans-Nerolidol & 31.61 & 0.30 \\
\hline 41 & Oxido de Cariofileno & 32.75 & 0.19 \\
\hline 42 & Guaiol & 33.03 & 16.32 \\
\hline 43 & Apiol & 33.44 & 5.41 \\
\hline 44 & Y-Eudesmol & 34.09 & 0.88 \\
\hline 45 & $\begin{array}{l}\text { 2-isopropil-5-metil-9-metileno-Biciclo[4.4.0] } \\
\text { dec-1-eno }\end{array}$ & 34.32 & 0.50 \\
\hline 46 & tau-Murolol & 34.38 & 0.21 \\
\hline 47 & a-Cadinol & 34.75 & 1.30 \\
\hline 48 & $\beta$-Eudesmol & 34.87 & 2.36 \\
\hline 49 & Bulnesol & 35.06 & 4.15 \\
\hline 50 & a-Bisabolol & 35.49 & 6.49 \\
\hline
\end{tabular}

$\operatorname{Tr}=$ Tiempo de retención, resultados en porcentaje de área relativa al pico 
Tabla 2. Composición química del aceite esencial de Mentha piperita L.

\begin{tabular}{|c|c|c|c|}
\hline Número & Nombre del compuesto (NIST08.L) & $\operatorname{tR}(\min )$ & $\begin{array}{l}\% \text { en la muestra } \\
\text { áreas relativas) }\end{array}$ \\
\hline 1 & $\beta$-Mirceno & 15.01 & 3.15 \\
\hline 2 & p-Cimeno & 16.30 & 0.14 \\
\hline 3 & $\beta$-trans-ocimeno & 16.49 & 4.58 \\
\hline 4 & Eucaliptol & 16.61 & 0.50 \\
\hline 5 & $\beta$-cis-ocimeno & 16.85 & 1.96 \\
\hline 6 & 4-Careno & 18.25 & 0.37 \\
\hline 7 & $\beta$-Linalol & 18.62 & 30.25 \\
\hline 8 & cis-Tagetona & 19.98 & 0.14 \\
\hline 9 & Mentona & 20.53 & 0.13 \\
\hline 10 & 3-Pinanona & 21.24 & 0.20 \\
\hline 11 & 4-Terpineol & 21.29 & 0.13 \\
\hline 12 & a-Terpineol & 21.70 & 5.75 \\
\hline 13 & cis-Geraniol & 22.38 & 1.29 \\
\hline 14 & $\beta$-Citral & 22.82 & 0.12 \\
\hline 15 & o-aminobenzoato de linalilo & 23.06 & 27.32 \\
\hline 16 & trans-Geraniol & 23.11 & 4.26 \\
\hline 17 & $\alpha$-Citral & 23.64 & 0.22 \\
\hline 18 & Acetato de Nerol & 26.05 & 2.92 \\
\hline 19 & Acetato de Geraniol & 26.58 & 5.48 \\
\hline 20 & $\beta$-Elemeno & 27.21 & 0.45 \\
\hline 21 & $\beta$-Cariofileno & 28.21 & 1.87 \\
\hline 22 & (Z)- $\beta$-Farneseno & 28.63 & 0.12 \\
\hline 23 & Germacreno D & 29.82 & 1.28 \\
\hline 24 & Elemol & 31.52 & 2.07 \\
\hline 25 & Óxido de cariofileno & 32.75 & 0.18 \\
\hline 26 & Ledol & 33.10 & 2.35 \\
\hline 27 & Apiol & 33.41 & 0.49 \\
\hline 28 & $\mathrm{Y}$-Eudesmol & 34.07 & 0.44 \\
\hline 29 & $\alpha$-Eudesmol & 34.86 & 0.48 \\
\hline
\end{tabular}

Tr=Tiempo de retención, resultados en porcentaje de área relativa al pico 
Los componentes bioactivos identificados en los aceites esenciales de las especies investigadas son en total 11 compuestos, principalmente hidrocarburos sesquiterpenicos y monoterpenicos: $\beta$-Mirceno, Eucaliptol, $\beta$-Linalol, cis-Tagetona, 4-Terpineol, $\beta$-Cariofileno, (Z)- $\beta$-Farneseno, Germacreno D,
Óxido de cariofileno, Apiol y y-Eudesmol. La actividad antioxidante de los aceites esenciales presento un mayor contenido de $7,21 \mathrm{mg} / \mathrm{mL}$ en el aceite esencial Mentha piperita L. y 4,95 $\mathrm{mg} / \mathrm{mL}$ en Lepechinia meyenii (Walp) Epling., como se presenta en la Tabla 3.

Tabla 3. Actividad antioxidante de los aceites esenciales por DPPH.

\begin{tabular}{|c|c|c|c|c|}
\hline Muestra & \multicolumn{3}{|c|}{ Actividad antioxidante } & Diferencia significativa \\
\hline Lepechinia meyenii (Walp) Epling & 4,95 & \pm & 0,06 & A \\
\hline Mentha piperita $\mathrm{L}$ & 7,21 & \pm & 0,21 & B \\
\hline
\end{tabular}

\section{Discusión}

El análisis de los componentes químicos en los aceites esenciales de las especies Lepechinia meyenii (Walp) Epling y Mentha piperita L., mostraron mayormente la presencia de los siguientes compuestos: Acetato de Mirtenilo, Guaiol, -Linalol y o-aminobenzoato de linalilo. El género Lepechinia, se caracteriza en general por los compuestos cariofeno, mirceno acetato de mirtenilo, guaiol y -pineno como componentes principales, mientras que el género Mentha contiene principalmente al -linalol, o-aminobenzoato de linalilo, -trans-ocimeno y mentona. De acuerdo a los resultados se identificaron como fracción principal a los componentes sesquiterpenos en Lepechinia y monoterpenoides en Mentha. Se sabe que los componentes monoterpenos y sesquiterpenos son metabolitos secundarios característicos de los aceites esenciales (25). Los sesquiterpenos y monoterpenoides tienen propiedades antioxidantes, anticonvulsivas, antiulcerosas, antiinflamatorias, antibacterianas y antidiabéticas terapéuticas $(26,27)$.
El mecanismo de acción general de los monoterpenos, como actividad antimicrobiana y antitusiva, se relaciona principalmente con su volatilidad, su hidrofobicidad, así como los aceites esenciales en su conjunto, determina su efecto sobre las estructuras de las células bacterianas con un efecto antimicrobiano posterior (28). Se ha demostrado que los monoterpenos ejercen actividades quimiopreventivas y quimioterapéuticas en modelos de tumores mamarios y por lo tanto pueden representar una nueva clase de agentes terapéuticos (27). En investigaciones anteriores compararon los principales componentes de los aceites esenciales obtenidos de especies Lepechinia spp. observándose monoterpenos y sesquiterpenos (25).

Se encontró una variación de componentes de las especies estudiadas en comparación al lugar de procedencia, donde se caracterizó un aceite esencial dominado por $\beta$-felandreno, canfeno, limoneno (29). Debido a la heterogenidad de los compuestos identificados en la especie Lepechinia no es 
posible establecer un patrón característico de compuestos para el género. El aceite esencial de Lepechinia conferta (30), y Lepechinia shiedeana (31), de Venezuela exhibieron Ledol con 24,2 a $28,9 \%$ respectivamente como compuestos principales. Para el caso de Lepechinia floribuna de Argentina, Borneol $21,4 \%$, $\beta$-Cariofileno $15,1 \%$ y acetato de ledilo $16,8 \%$ fueron los compuestos principales; sin embargo, para la misma especie recolectada en Bolivia, fue acetato de bornilo $12,3 \%$, $\beta$-cariofileno $9,0 \%$ y canfeno $5,7 \%$ como compuestos principales (32).

En el aceite esencial de Lepechinia meyeni (Walp) Epling los monoterpenos constituían la fracción más importante con $61 \%$ seguidamente con $40 \%$ seguido de sesquiterpenos oxigenados (32). Otra especie endémica del género Lepechinia presente en Chile (35), reportó una composición particularmente rica en sesquiterpenos (50.6\%), pero con porcentajes similares de componentes oxigenados e hidrocarburos $(26,4 \%)$ y $(24,2 \%)$ respectivamente. En general los sesquiterpenoides son la principal familia de componentes en los aceites esenciales de Lepechinia spp donde se reporta una gran variación entre los componentes principales, aunque se recolectan en el mismo lugar (31). De acuerdo a estudios de aceites esenciales de las hojas de menta que crecen en la Unicamp (Brasil) se caracterizan por la predomino del alcohol monoterpenico (linalol) con una tasa de (51\%) seguido de carvona (23,42\%) (37). En otras investigaciones los compuestos dominantes del género Mentha de Irán fueron -Terpineol y oxido de pipertitinona (38). Varios artículos han informado de que la composición química de los aceites esenciales de Mentha difiere según los países o regiones en el mismo estado. Estas variaciones parecen depender de varias razones como cambios climáticos, externos medio ambiente y otros factores como el método y periodo de extracción, partes recolectadas de la planta, riego tectónicas y fenológicas. $(39,40)$.

En cuanto a las bioactividades de estos compuestos el p-cimerno, según Cristiani y colaboradores han informado de la actividad antimicrobiana de cuatro monoterpenos entre ellosel (p-cimeno, $y$-tirpineno, carvacrolytimol) contra la bacteria Gram_positiva S. aureus y la bacteria Gram-negativa E.coli. concluyendo que el timol era considerablemente más toxico contra S. aureus que los otros tres terpenos, mientras que el carvacrol y el p-cimeno eran más activos contra E. coli. (41). El compuesto germadeno $\mathrm{D}$ compuesto que tiene tres dobles enlaces ricos en electrones, demostró una capacidad para eliminar aniones de radicales superixido (42). En los aceites esenciales que se encuentran en concentraciones más altas y relacionados con la actividad antimicrobiana son compuestos fenólicos como linalo, sabineno, mentol, mirceno y canfeno (43). Los sesquiterpenos tienen propiedades antiinflamatorias y antialérgicas. Las actividades antiinflamatias de algunas plantas medicinales se deben a la presencia de una o más lactonas sesquiterpenicas (44).

Con respecto a la actividad antioxidante de los aceites esenciales ambas especies presentan significativamente presencia de actividad antioxidante, de acuerdo investigaciones se sabe que los aceites esenciales con componentes dominantes como pulegona (44), mentona y epóxido de cis-piperitona $(45,46)$, exhiben antioxidantes (47). Los compuestos timol, eucaliptol, mentol y carvacrol pertenecen a compuestos antioxidantes capaces de prevenir el estrés oxidativo (48). De acuerdo a los resultados 
obtenidos, observamos la coincidencia con los informes investigativos en los que el poder antioxidante in vintro de los aceites esenciales fue evaluado y vinculado a los principales monoterpenos oxigenados incluyendo mentol, mentona, acetato de mentilo y 1,8-cineol $(49,50)$. En otros estudios sobre el aceite esencial de especies de Mentha pipireta L. de menor compuesto químico que contiene moléculas en el grupo metileno activo, tales como terpinoleno, $\alpha-y$ y- Terpineno, fueron también incluidos y reconocidos por su poderosa acción antioxidante que es equivalente al estándar positivo (vitamina $\mathrm{E} \mathrm{O}$ a-tocoferol) (51).

\section{CONCLUSIONES}

En el estudio se logró determinar el perfil químico y actividad antioxidante de los aceites esenciales de las especies de Lepechinia meyeni (Walp) Epling y Mentha pipireta L. Las propiedades físicas de ambas especies presentaron rangos de calidad para aceites esenciales. Se detectaron y cuantificaron un total de 61 compuestos químicos para el aceite esencial de Lepechinia meyenii (Walp) Epling, siendo los hidrocarburos sesquiterpenicos la fracción principal de (55,73\%). Sin embargo en la especie Mentha piperita L. se detectaron y cuantificaron 36 constituyentes, siendo los compuestos monoterpenoides principales. Las propiedades físicas de ambas especies presentan ron rangos de calidad para aceites esenciales. La abundancia en monoterpenos conducen a actividades antioxidantes, encontrándose en el estudio mayor presencia de antioxidantes en la especie de Mentha piperita L. Además se concluye que los aceites esenciales de Lepechinia meyenii (Walp) Epling y Mentha piperita L. presentaron una buena actividad antioxidante siendo posibles candidatas por sus actividades antimicrobianas, antioxidantes, anticonvulsivas, antiulcerosas, antiinflamatorias, antibacterianas y antidiabéticas terapéuticas de mucho interés para las industrias con un enfoque farmacológico y alimentario especifico.

\section{REFERENCIAS BIBLIOGRÁFICAS}

1. Vásquez $P$, Cojean $S$, Rengifo $E$, Suyyagh AS, Amasifuen G, Pomel S, Cabanillas B et al. Antiprotozoal activity of medicinal plants used by lquitos-Nauta road communities in Loreto (Peru). J. Ethnopharmacol 2018; 210: 372385. [Links]

2. Fejér J, Grul'ová D, De Feo V, Ürgeová E, Obert B, Pret'ová A. Mentha x piperita L. nodal segments cultures and their essential oil production. Ind. Crops Prod. 2018; 112: 550555. [Links]

3. Ertas A, Boga M, Yilmaz MA, Yesil Y, Tel G, Temel H, Hasimi N, Gazioglu I, Ozturk M, Ugurlu P. A detailed study on the chemical and biological profiles of essential oil and methanol extract of Thymus nummularius (Anzer tea): Rosmarinic acid. Ind. Crops Prod. 2015; 67: 336-345. [Links]

4. Mamadalieva NZ, Youssef FS, Ashour ML, Sasmakov SA, Tiezzi A, Azimova SS. Chemical composition, antimicrobial and antioxidant activities of the essential oils of three Uzbek Lamiaceae species. Natural Product Research 2019;33(16): 2394-2397. [Links]

5. Burt S. Essential oils: Their antibacterial properties and potential applications in foods a review. Int. J. Food Microbiol. 2004; 94(3): 223- 253. [Links]

6. Božovic M, Ragno R. Calamintha nepeta (L.) Savi and its main essential oil constituent pulegone: Biological activities and chemistry. Molecules 2017; 22(2): 1-50. [Links]

7. Preedy V. Essential oils in food presservation, flavor and safety. Academic Press is an imprint of Elsevier, Amsterdam 2016;932 pp. [Links] 
8. Teles S, Pereira JA, Santos $\mathrm{CH}$, Menezes RV, Malheiro R, Lucchese, AM, Silva F. Effect of geographical origin on the essential oil content and composition of fresh and dried Menthaxvillosa Hudson leaves. Ind. Crops Prod. 2013;46: 1-7. [Links]

9. Boukhatem MN, Amine FM, Kameli A, Saidi F, Walid K, Mohamed, SB. Quality assessment of the essential oil from Eucalyptus globulus Labill of Blida (Algeria) origin. Int. Lett. Chem. Phys. Astron. 2014; 36: 303315. [Links]

10. Pandey BP, Thapa R, Upreti A. Chemical composition, antioxidant and antibacterial activities of essential oil and methanol extract of Artemisia vulgaris and Gaultheria fragrantissima collected from Nepal. Asian Pac. J. Trop. Med. 2017; 10: 952-959. [ Links ]

11. Van HS, Raes K, Van DM, SampersI. The effect of cinnamon, oregano and thyme essential oils in marinade on the microbial shelf life of fish and meat products. Food Control 2016; 68: 3039. [Links]

12. Ju J, Xu X, Xie Y, Guo Y, Cheng Y, Qian H, Yao W. Inhibitory effects of cinnamon and clove essential oils on mold growth on baked foods. Food Chem. 2018; 240: 850-855. [Links]

13. Başer $\mathrm{KH}$, Buchbauer G Eds. Handbook of Essential Oils: Science, Technology and Applications. Boca Raton. CRC Press/Taylor \& Francis. 2010: 994 pp. [Links]

14. d'Acampora ZB, Dugo P, Dugo G, Mondelo. Analysis of essential oils. En: Handbook of Essential oils: Science, Technology and Applications. Can Baser, K., ed. Boca Raton. CRC Press/Taylor \& Francis. 2010

15. Adams T, Taylor, S. Safety evaluation of essential oils: a constituent-based approach. En: Handbook of Essential oils: Science, Technology and Applications. Can Baser, K., ed. Boca Raton. CRC Press/Taylor \& Francis. 2010:185-208 pp. [Links]

16. Escuder B, Torres R, Lissi E, Labbé C, Faini F. Antioxidant Capacity of Abietanes from Sphacele salviae. Nat. Prod. Lett. 2002; 16(4):277-281
17. Maier L, Lineros T, Oberpaur C, Aracena D, Délano G. Antimicrobial Effect of White Sage (Sphacele salviae (Lindl.) Briq.) Leaf Extract on Gram-Positive and GramNegative Bacteria. Agro. Sur) 2014; 42(3): 47-54.

18. Labbé C, Faini F, Calderón D, Molina J, Arredondo S. Variations of Carnosic Acid and Carnosol Concentrations in Ethanol Extracts of Wild Lepechinia salviae in Spring (2008-2011). Nat. Prod. Commun. 2014; 9(10):1413-1416. 6.

19. Montenegro G, Timmermann, B. Chile Nuestra Flora Útil: Guía de Plantas de Uso Apícola, En Medicina Folklórica, Artesanal y Ornamental, Primera Ed.; Chile Universidad Catoilica de, Ed.; Santiago, Chile. 2000.

20. Gianluca, et al. Fitoquimica de tres Lamiaceae escuatorianas: Lepechinia heteromorpha (Briq.) Eplig, Lepechinia rádula (Benth.) Epling y Lepechinia paniculata (Kunth) Epling. Departamento de química y ciencia, Universidad Tecnica particular de Loja (UTPL). 2018: https://doi.org/10.3390/plants8010001

21. McKay DL, Blumberg JB. A review of the bioactivity and potential health benefits of peppermint tea (Mentha piperita L.). Phytother Res. 2006; 20:619-33.

22. Calo JR, Crandall PG, O'Bryan CA, Ricke SC. Essential oils as antimicrobials in food systems - A review. Food Control 2015; 54:111-19.

23. Lawrence BM. 2007. The composition of commercially important mints. En: Lawrence BM, 1 st edition, editor. Mint. The genus Mentha. Boca Raton, FL.: CRC Press, Taylor and Francis Group: 2007; 217-324 pp.

24. Adams RP. Identification of Essential Oil Components by Gas Chromatography/ Quadrupole Mass Spectroscopy. Carol Stream, IL. Allured Publishing, Co 1 st edition: 2000; 804 p

25. Ramírez J, Gilardoni $G$, Jácome $M$, Montesinos J, Rodolfi $M$, Guglielminetti, ML, Guglielminetti ML Cagliero C, Bicchi C, Vidari G. Composición química, análisis enantiomérico, evaluación sensorial AEDA y actividad antifúngica del aceite esencial de la planta ecuatoriana Lepechinia mutica Benth 
(Lamiaceae). Chem. Biodiversidad. 2017;14. [Google ] [CrossRef] [PubMed]

26. Dick AJ, Starmans HHN. Extraction of secondary metabolites from plant material: A review. Trends in Food Science and Technology. 1996;7(6):191-197

27. Shuaib A, Rohit A, Piyush MA. Review article on essential oils. Journal of Medicinal Plants Studies. 2016;4(3):237-240

28. Dragomanova S, Tancheva L, Georgieva M. A review: Biological activity of myrtenal and some myrtenalcontaining medicinal plant essential oils. Scripta Scientifica Pharmaceutica. 2018;5(2):22-33

29. Malagón O, Vila R, Iglesias J, Zaragoza T, and Cañigueral S. Composition of the essential oils of four medicinal plants from Ecuador, Flavour Frag. J. 2003; 18, 527-531.

30. Borges R, Rojas LB, Cegarra JÁ, and Usubillaga A. Study of the essential oils from the leaves and flowers of Lepechinia conferta (Benth) Epl, Flavour Frag. J. 2006; 21:155-157

31. Rojas LB, Usubillaga A, Cegarra JA, Borregales E, and Carrero S. Composición química y actividad Antimicótica del aceite esencial de la Lepechinia schiedeana (Schlecht) Vatke, Rev. Fac. Farm. 2004; 46:27-30.

32. Arze JBL, Collin G, Garneau FX, Jean FI, and Gagnon H. Essential Oils from Bolivia. VI. Lamiaceae: Lepechinia graveolens (Reg.) Epling L. floribunda (Benth.) Epling, and L. meyeni (Walp.) Epling, J. Essent. Oil Res. 2009; 21:36-40.

33. Valenzuela L, Vila R, Cañigueral S., Adzet, T. The Essential Oil of Sphacele chamaedryoides. Planta Med. 1992; 58(03):273-274.

34. Sartoratto $A$, Machado LM, Delarmelina C, Figueira GM, Duarte MCT, Rehder VLG, Composition and antimicrobial activity of essential oils from aromatic plants used in Brazil, Brazilian Journal of Microbiology, 2004:35:275-280. https://doi.org/10.1590/ S1517- 83822004000300001
35. REZAei MB, Taghizadeh $M$, Astanch SA., Rasooli I, Biochemical activities of Iranian Mentha piperita L. and Myrtus communis L. essential oils, Phytochemistry. 2006; 67:12491255.

36. Allali H, Chikhi I, DIB Mea, Muselli A, Fekih $\mathrm{N}$, Meliani N, Costa J, Antioxidant activity and chemical analysis of Mentha spicata cultivated from west northern region of Algeria by headspace solid phase microextraction and hydro-distillation, Natural Products, 2013; 9: 258-63,

37. Laggoune $S$, Öztürk $M$, Erol E, Duru ME, Abaza I, Kabouche A, Kabouche Z, Chemical composition, antioxidant and antibacterial activities of the essential oil of Mentha spicata L. from Algeria, Journal of Materials and Environmental Sciences. 2016; 7:4205-4213. https://doi.org/10.1016/j. foodchem.2007.03.059

38. Korosh AR, Juliani HR, Zygaldo JA. Bioactivity of essential oils and their components. In: Berger RG, editor. Flavours and Fragrances. Chemistry, Bioprocessing and Sustainability. Berlin: Springer; 2005.

39. Gouvea FDS, Rosenthal A, Ferreira EHR. Plant extract and essential oils added as antimicrobials to cheeses: A review. Ciencia Rural, Santa Maria. 2017;47:1-9

40. Wang $L$, Well CL. Recent advances in extraction of nutraceuticals from plants. Trends in Food and Sciences technology. 2006; 17:300-312.

41. Sutour S, Bradesi P, Rocca SD, Casanova J, Tomi F. Chemical composition and antibacterial activity the essential oil from Mentha suave olensssp. Insularis (Req.) Greuter.FlavourFrag.J. 2008; 23,107-114.

42. Oumzil $H$, Ghoulami $S$, Rhajaoui M, Ilidrissi, A, Fkih-Tetouani $S$, Faid $M$, Benjouad $A$. Antibacterial and antifungal activity of essential oils of Mentha suave olens. PhytotherRes. 2002; 16:727-731 
43. Kasrati A, Alaoui-Jamali C, Bekkouche K, Spooner-Hart R, Leach D, Abbad A. Chemical characterization and insecticidal properties of essential oils from differentwild populations of Mentha suave olens subsp. timija(Briq.) Harleyfrom Morocco.Chem. Biodivers. 2015; (12):823-831

44. Brahmi $F$, Abdenour A, BrunocM, Silviac P, Alessandrac P, Daniloc F, DrifaaYG, et al. Chemicalcompositionandinvitroantimicrobial, insecticidal and antioxidantactivities of the essential oils of Mentha pulegiumL. and Mentha rotundifolia (L.)Hudsgrowingin Algeria. Ind. CropsProd. 2016a; 88:96-105.

45. Qasim Barkat $M$, Khalid Mahmood $H$. Detección fitoquímica y antioxidante de Zingiber officinale, Piper Nigrum, Rutag Raveolanes y Carum Carvi y su efecto sobre la actividad del tracto gastrointestinal. Matrix
Sci. Medica 2018;(2):09-13.[Google Académico] [CrossRef]

46. Yang SA, JEon SK, Lee EJ, Shim CH, Lee IS. Comparative study of the chemical composition and antioxidant activity of six essential oils and their components, Natural Product Research. 2010; 24: 140-151, https:// doi.org/10.1080/14786410802496598

47. Rozza AL, DE Faria FM, Brito ARS, Pellizzon $\mathrm{CH}$. The gastroprotective effect of menthol: involvement of anti-apoptotic, antioxidant and anti-inflammatory activities, PloS one, 9, 2014. https://doi.org/10.1371/journal.pone.0086686

48. Ruberto G, Baratta MT, Antioxidant activity of selected essential oil components in two lipid model systems, Food Chemistry, 2000; 69:167-174. https://doi.org/10.1016/S03088146(99)00247-2 